\title{
Anatomical dissection of a cadaver with congenital scoliosis
}

\author{
A. Stevenson ${ }^{1}$, S. McCarthy², J. Kalmey², R. Kulesza² \\ ${ }^{1}$ Department of General Surgery, Geisinger Wyoming Valley, PA, United States \\ ${ }^{2}$ Department of Anatomy, Lake Erie College of Osteopathic Medicine, PA, United States
}

[Received 27 September 2013, Accepted 15 November 2013]

\begin{abstract}
Congenital scoliosis is a developmental anomaly involving poorly formed or fused vertebral segments resulting in an abnormal lateral curvature of the vertebral column and is often accompanied by significant rotational defects. Despite abundant literature on causes, diagnosis and treatment of scoliosis, little attention has been given to impacts of this condition on the musculoskeletal system beyond the bony defects. This report describes the detailed, layer-by-layer dissection of the superficial and deep back musculature and examination of the axial skeleton of a 47-year-old male with severe congenital scoliosis. The subject presented with both cervico-thoracic and thoraco-lumbar scoliotic curves. Dissection of the back muscles revealed notable asymmetry in the superficial muscles and marked atrophy of the deep back muscles on the left side. Examination of the axial skeleton revealed numerous bones which were abnormally porous, reduced thoracic volume, attenuated intercostal spaces on the left side and 2 separate fusion deficits, including an unsegmented bar spanning 6 vertebral segments. (Folia Morphol 2014; 72, 3: 389-394)
\end{abstract}

Key words: dysmorphology, unsegmented bar, vertebra

\section{INTRODUCTION}

Scoliosis is a common musculoskeletal disorder, affecting approximately $4 \%$ of the population and is characterised by an excessive lateral curvature of the vertebral column [18]. Scoliosis is traditionally classified as neuromuscular, congenital or idiopathic. Neuromuscular scoliosis can result from upper or lower motor neuron disease, muscular dystrophy or other myopathies [11]. Congenital scoliosis (CS) is a less common developmental malformation of the vertebral column (1:1000) [20] which results from abnormal segmentation of embryonic sclerotome masses [17] and is commonly accompanied by rotational displacement $[7,11,13,19]$. The characteristic lateral curvature of CS is attributed to poorly formed or unsegmented vertebra (hemivertebra or unilateral unsegmented bars [UB], respectively). CS involving UB can result in severe curves which extend well beyond the bony defects and such defects can further impact volume of the thoracic cage and respiratory movements $[6,15]$. Although there is extensive literature on causes, severity and management of scoliosis, there are no reports on the alterations of the soft tissue surrounding the vertebral defects or detailed descriptions of dissected specimens (but see Gillaspy and Van Renterghem [8], who provide a post-mortem study of the vertebral column from a patient with (S). Herein is described a detailed, layer by layer dissection of back muscles and examination of the axial skeleton from a 47-year-old male with severe CS.

\section{CASE REPORT}

A 47-year-old male cadaver was donated to the school's anatomy laboratory for dissection. The cause 

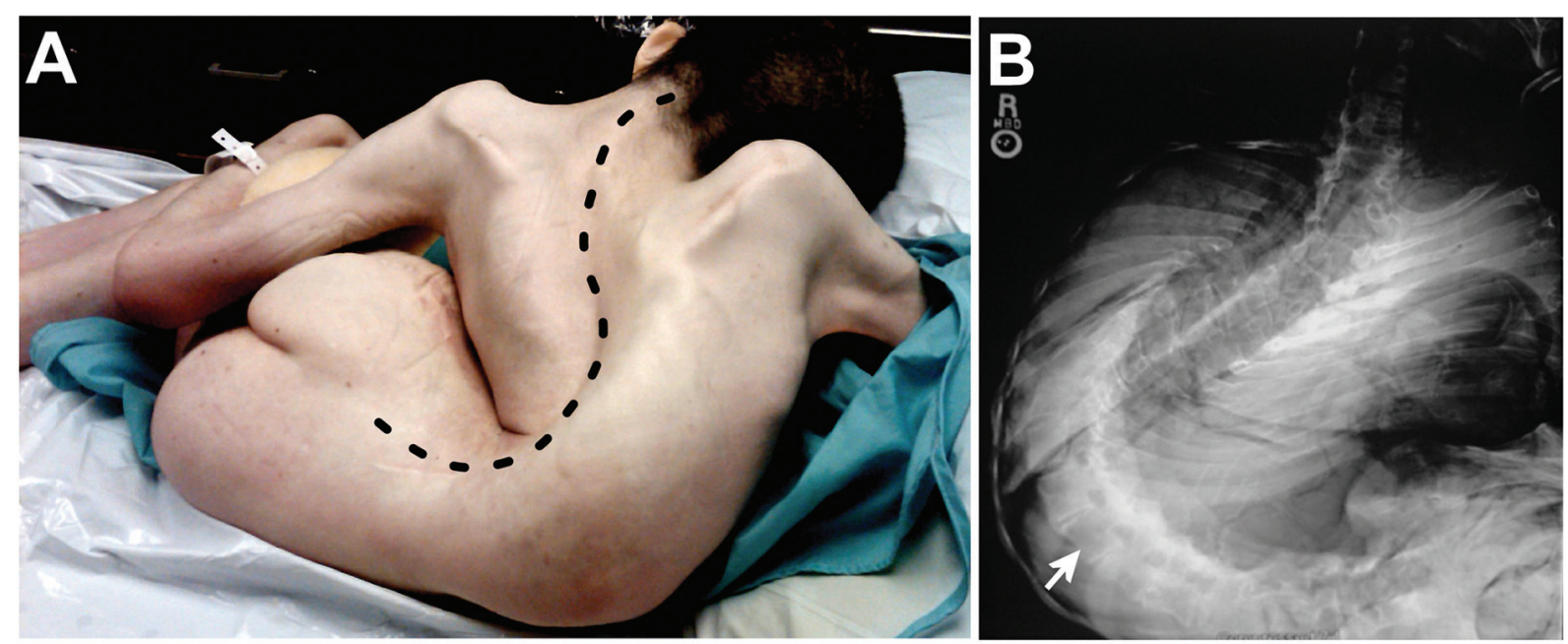

Figure 1. Physical examination and imaging. The subject presented to our morgue as shown in panel A. An obvious S-shaped curve is indicated by the dashed line. An AP radiograph of his vertebral column (B) illustrates multiple curves and a significant lumbar rotational defect (arrow).

of death was documented as respiratory failure secondary to aspiration pneumonitis. The patient was non-ambulatory with a medical history significant for lumbar spina bifida and mental retardation of unknown severity. Medical documentation of the patient's scoliosis progress was unavailable.

On gross appearance, the cadaver was cachectic with obvious severe scoliosis (Fig. 1A). There was a rightward side bending at the cervico-thoracic junction and a left-ward side bending curve involving the inferior thoracic and superior lumbar segments (see dashed line in Fig. 1A). Surgical scars were present at the lumbosacral junction and hip from previous surgical procedures (i.e. surgical correction of spina bifida). The subject did not show any outward signs of other congenital anomalies and there were no observed cutaneous abnormalities (e.g. patches of hair, red nevus or telangiectasis) of the skin overlying the vertebral column. Postmortem radiographs (Fig. 1B) further documented both a cervico-thoracic curve and a thoraco-lumbar curve accompanied by a significant rotational defect (see arrow in Fig. 1B). Severity of the curves was estimated by the Cobb method [4]. The cervico-thoracic curve (T2-C6) revealed an angle of $16^{\circ}$ with no rotation while the thoraco-lumbar curve (L2-T8) measured $131^{\circ}$ with significant rotation (4+ in the middle of the curve).

Removal of the skin and superficial fascia from the back revealed the trapezius and latissimus dorsi muscles (see arrows in Fig. 2A). Closer examination of the thoracic spine revealed significant misalign- ment of thoracic spinous processes (see asterisk in Fig. 2B) and marked asymmetry in the trapezius, latissimus dorsi (Fig. 2A) and rhomboids (not shown). Reflection of the superficial back muscles revealed the full extent of the erector spinae group (Fig. 2C), which were arranged in a tortuous manner. On the left side this muscle was severely atrophied and in many regions existed as only tendinous insertions (see white arrow in Fig. 2C). On the right side, the erector spinae had a typical fleshy muscle belly but because of the thoraco-lumbar curvature, the tendons of the iliocostalis muscle inserted in a radial manner (see black arrows in Fig. 2C). Removal of the erector spinae muscles revealed the multifidus (Fig. 2D). Again, on the left side the multifidus was atrophied (see white arrow in Fig. 2D) and could not be identified superior to the tenth thoracic spinous process. On the right, the multifidus was more typical in appearance (see black arrow in Fig. 2D) but at many levels existed only as tendinous slips. The remaining deep back (rotatores, semispinalis, levator costarum and splenius) and suboccipital muscles were typical and relatively symmetric (not shown). Beyond the effects of diminished thoracic volume, there were no obvious congenital anomalies affecting the thoracic and abdominal organs and the organs of the body cavity appeared normal. Removal of the brain revealed an enlarged foramen magnum and herniation of the cerebellar tonsils inferior to the foramen magnum, consistent with a Chiari type 1 malformation. 

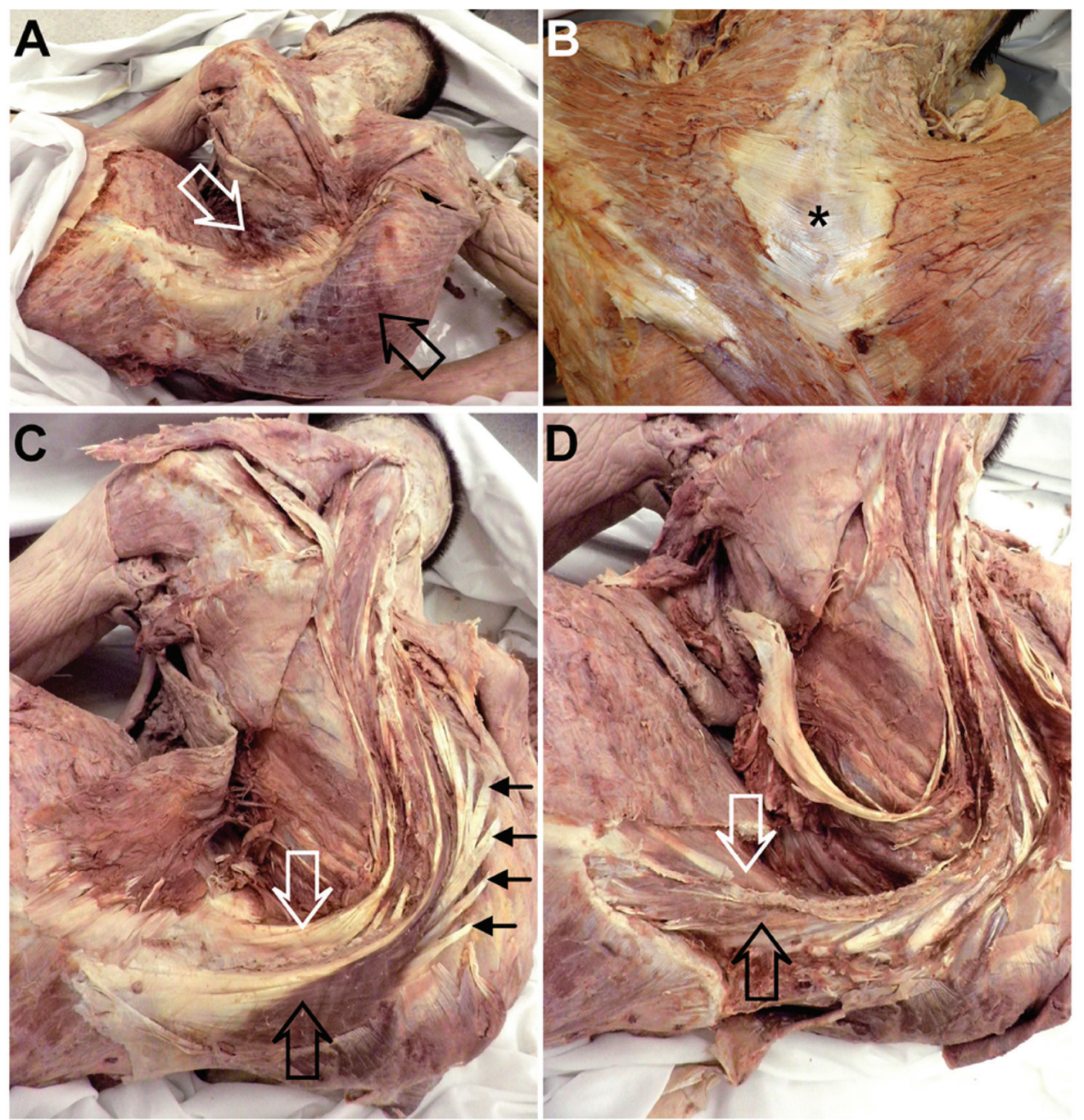

Figure 2. Superficial and deep back muscles. Examination of the superficial back muscles $(\mathbf{A})$ revealed marked asymmetry in the trapezius and latissimus dorsi (arrows); B. Closer examination revealed offset spinous processes (asterisk indicates vertebra prominens and fusion defect, see Fig. 4B); C. The erector spinae were asymmetric: the left side (white arrow) was atrophied, while the right side (black arrow) had normal bulk, but the iliocostalis tendons inserted in a radial manner (arrows); D. The multifidus muscle was also asymmetric: the left side was atrophied (white arrow) while the right side was more typical (black arrow).

The remaining soft tissue was removed from the vertebral column, thoracic cage and pelvis to more effectively examine the bony defects. In general, all of the bones appeared to be especially porotic, and this was most notable in the manubrium, sacrum and iliac crests (Fig. 3). Examination of the articulated pelvis revealed marked asymmetry between the two pelvic bones (Fig. 3B). Further inspection revealed that the left acetabulum was malformed and there were bony abnormalities observed at the right and left iliopectineal eminences that suggested compression defects (Fig. 3B). Further, the sacrum was angled such that the right anterior sacral foramina were anterior and inferior to those on the left (Figs. 3B, C) and the foramina of the fourth sacral segment were nearly imperceptible (see white arrow in Fig. 3C). Examination of the thoracic cage revealed a significant asymmetry in the size of the pulmonary cavities (Fig. 4A). Notably, on the left side the average intercostal space measured $0.3 \mathrm{~cm}$ while on the right side the intercostal spaces averaged $2.75 \mathrm{~cm}$. Further, on the left side the last rib was apposed to the iliac crest while on the right side this interval measured $20.32 \mathrm{~cm}$ (see Fig. 1B). Examination of the vertebral column revealed multiple bony defects and an additional lumbar vertebra. At C4-C6 there was notable growth of osteophytes from the right side of the vertebral bodies. At C7 and T1, there was a fusion defect 

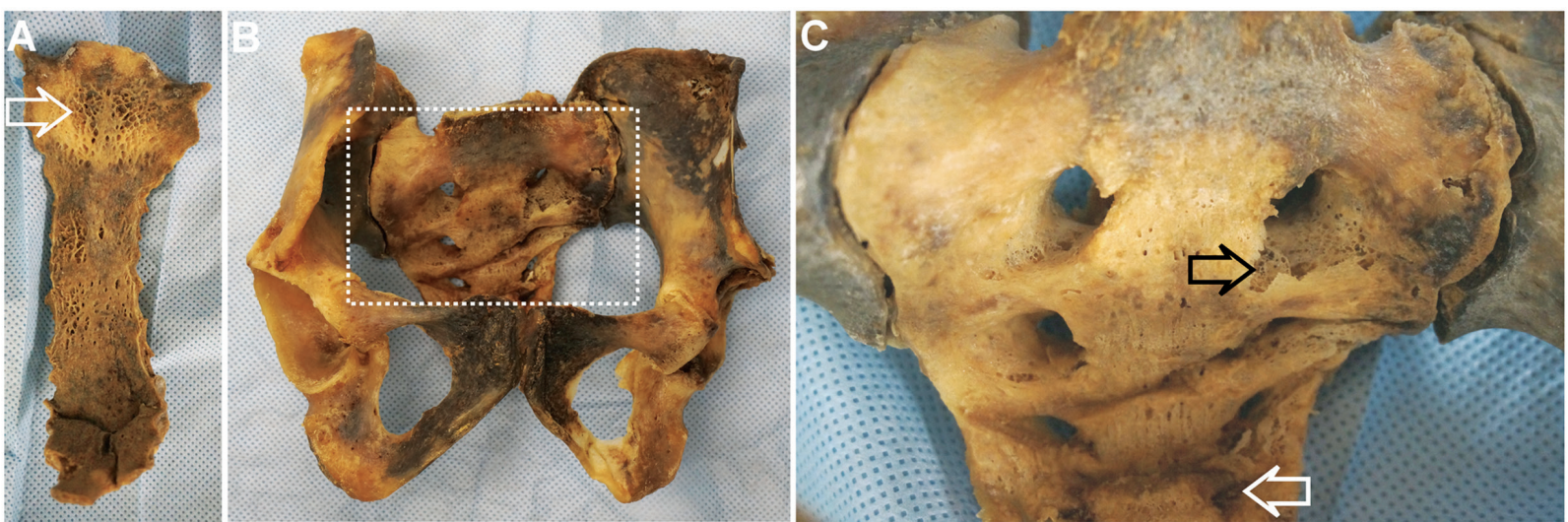

Figure 3. Porosity of the sternum and sacrum. The posterior surface of the sternum was notably porous (white arrow in panel A). The articulated pelvis displayed marked asymmetry (B; inset indicating area of panel C); C. The anterior surface of the sacrum also demonstrated porosity (black arrow) and asymmetrical sacral foramina (white arrow, nearly closed anterior sacral foramen).

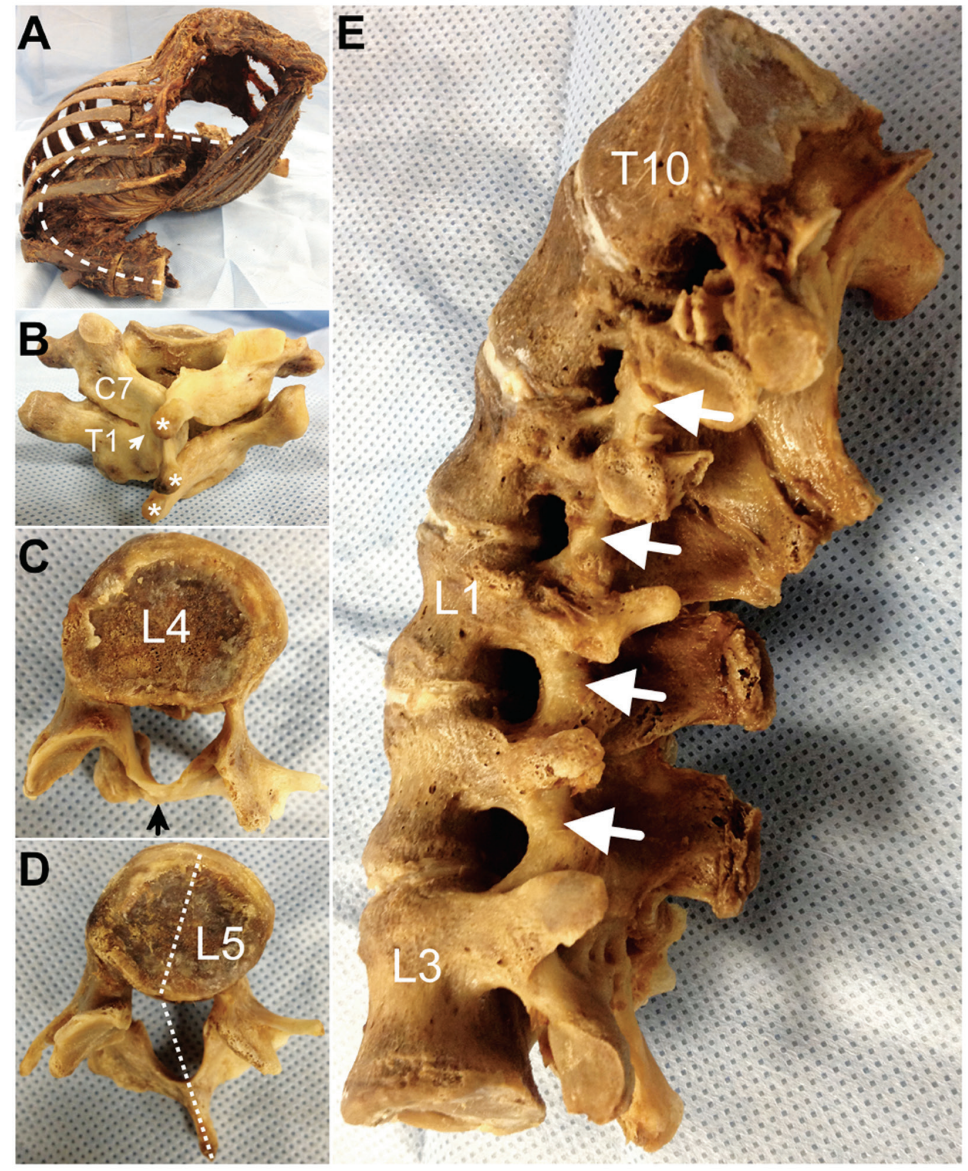

Figure 4. Inspection of the axial skeleton. Examination of the thoracic cage revealed significant asymmetry in the size and shape of the pulmonary cavities (A). The dashed line in panel A indicates the thoraco-lumbar curve; B. There was a fusion defect involving C7 and T1: the lamina of these vertebrae were fused on the left side (arrow) and gave rise to 3 spinous processes (asterisks). The L3 and L4 vertebrae lacked spinous processes (arrow in C) and the L5 and L6 posterior arches were angulated to the right side of the vertebral body (dashed lines in panel D). The 6-segment spanning unsegmented bar is shown in panel $\mathbf{E}$. The articular pillars were fused on the left side such that no synovial articulations were found (arrows in panel E). 
(Fig. 4B). On the right side, the lamina of both vertebrae ended as separate spinous processes; however, on the left side, both lamina coalesced to form a fused spinous process (see arrow in Fig. 4B; see also asterisk in Fig. 2B). As a result, three spinous processes were formed between these two vertebrae (see asterisks in Fig. 4B). From T3 to T9, the spinous processes and lamina were asymmetric and were directed to the left of the midline. The L3 and L4 vertebrae lacked spinous processes (see arrow in Fig. 4C) and the spinous processes and lamina of L4-L6 were asymmetric and were oriented to the right of the midline (see dashed line in Fig. 4D). The largest bony defect involved T10-L3, which included an UB involving the articular processes (T10-L3) and lamina (T12-L3; arrows in Fig. 4E). Thus, there were no facet joints on the left side from T10 to L3. Additionally, there was an incomplete facet joint present on the left between T10 and T11. Finally, there was partial fusion of the facet joint between L2 and L3 on the right side.

\section{DISCUSSION}

This case study describes the detailed dissection of the superficial, intrinsic back muscles, thoracic cage, vertebral column and pelvis from a 47-year-old male with severe CS due to multiple bony defects. Although detailed computed tomography reconstructions might be able to provide resolution adequate to make some similar observations, detailed dissection permitted evaluation of individual muscle layers and attachments and drying the bones permitted unobstructed examination of bony defects and subtle bony changes (i.e. porosity). In this case of severe scoliosis there was marked asymmetry of the superficial back muscles and significant atrophy of the intrinsic back muscles on the concave side. Quantification of the intercostal spaces revealed a significant impact of vertebral curvature on the thoracic cage. Specifically, we identified reduced thoracic volume and nearly obliterated intercostal spaces on the left side.

The bones from this cadaver appeared to be especially porous suggesting a lower bone mineral density (BMD). While BMD was not measured in this specimen, scoliosis is known to be associated with lower BMD [3, 5, 22]. Specifically, Zhu et al. [22] measured the BMD of individuals with $C S$ at the femoral neck and L2-4 spine and reported a significantly lower BMD compared to age matched controls. Further, it is well understood that mechanical load correlates with BMD [9]. The severity of the scoliotic curves in this cadaver (and his history) indicated severely limited mobility, resulting in decreased weight-bearing on his vertebral column and pelvis likely contributing to decreased BMD.

This specimen had two major vertebral fusion anomalies, including a large UB extending from $\mathrm{T} 10$ to L3. Thus, the UB in this patient extended over 6 vertebral segments. UB anomalies span, on average, 3 vertebral segments but have been observed to extend over 8 vertebral segments [15]. Further, UBs are known to have significant curve progression and poor prognosis $[14,16,19,21]$. It has been suggested by Lonstein et al. [12] that previous reports of decreased glycosaminoglycan levels at the level of the apex of the scoliotic curve likely contribute to this progressive worsening of curvatures. Finally, progression and prognosis of CS with an UB is likely attributed to the fact that regions of UBs lack growth plates and these regions therefore do not grow longitudinally [15].

According to previous work, malformations of the ribs, chest cavity, and scapulae are often a common finding with severe scoliosis $[1,2,8,10]$. Additionally, it has been suggested that when spinal deformities are severe, thoracic insufficiency syndrome is often present as the thorax is unable to support normal respiratory movements [6]. It appears that the observed asymmetry in the thoracic cavities contributed significantly to the patient's respiratory failure. Based on these observations of asymmetry and atrophy of superficial and deep back muscles, it appears that the musculo-fascial system may require special attention in treatment of CS and that therapies directed towards musculoskeletal structures beyond observed bony defects might provide an avenue to address and/or improve respiratory function in patients with CS.

\section{ACKNOWLEDGEMENTS}

The authors would like to thank the donor and his family for their generous donation.

\section{REFERENCES}

1. Athanasios TI, McMaster MJ (2005) Congenital Anomalies of the Ribs and Chest Wall Associated with Congenital Deformities of the Spine. Am J Bone Joint Surg, 87A: 2523-2536. 
2. Campbell R (2009) Spine deformities in rare congenital syndromes. Spine, 34: 1815-1827.

3. Cheng JC, Guo X (1997) Osteopenia in adolescent idiopathic scoliosis. A primary problem or secondary to the spinal deformity? Spine, 22: 1716-1721.

4. Cobb JR (1948) Outline for the study of scoliosis. In: The American Academy of Orthopedic Surgeons Instructional Course Lectures. Vol. 5. Ann Arbor, Edwards, MI.

5. Cook SD, Harding AF, Morgan EL, Nicholson RJ, Thomas KA, Whitecloud TS, Ratner ES (1987) Trabecular bone mineral density in idiopathic scoliosis. J Pedatric Orthopaedics, 7: 168-174.

6. Day GA, Upadhyay SS, Ho EK, Leong JC, Ip M (1994) Pulmonary function in congenital scoliosis. Spine, 19: 1027-1031.

7. Giampietro PF, Blank RD, Raggio $C L$, Merchant $S$, Jacobsen S, Faciszewski T, Shukla SK, Greenlee AR, Reynolds C, Schowalter DB (2003) Congenital and idiopathic scoliosis: clinical and genetic aspects. Clin Med Res, 1: 125-136.

8. Gillaspy C, Van Renterghem O (1954) An anatomical description of a specimen of extreme scoliosis with associated variations. Anat Rec, 120: 545-554.

9. Going SB, Laudermilk, M (2009) Osteoporosis and strength training. Am J Lifestyle Med, 3: 310-319.

10. Grivas TB, Burwell GR, Vasiliadis ES, Webb JK (2006) A segmental radiological study of the spine and rib-cage in children with progressive infantile idiopathic scoliosis, Scoliosis, 1: 1748-1761.

11. Janicki JA, Alman B (2007) Scoliosis: review of diagnosis and treatment. Paediatr Child Health Nov, 12: 771-776.

12. Lonstein J, Winter R, Bradford D, Ogilvie J (1995) Moe's textbook of scoliosis and other spinal deformities. $3^{\text {rd }} \mathrm{Ed}$. W.B. Saunders, Philadelphia, PA.
13. Lopez-Sosa F, Guille JT, Bowen JR (1995) Rotation of the spine in congenital scoliosis. J Pediatr Orthop, 15: 528-534.

14. McMaster MJ (1998) Congenital scoliosis caused by a unilateral failure of vertebral segmentation with contralateral hemivertebrae. Spine, 23: 998-1005.

15. McMaster MJ, McMaster ME (2013) Prognosis for congenital scoliosis due to a unilateral failure of vertebral segmentation. J Bone Joint Surg Am, 95: 972-979 .

16. McMaster MJ, Ohtsuka K (1982) The natural history of congenital scoliosis. A study of two hundred and fifty-one patients. J Bone Joint Surg Am, 64: 1128-1147.

17. Pourquié $O$ (2011) Vertebrate segmentation: from cyclic gene networks to scoliosis. Cell, 145: 650-663.

18. Rogala EJ, Drummond DS, Gurr J (1978) Scoliosis: incidence and natural history. A prospective epidemiological study. J Bone Joint Surg Am, 60: 173-176.

19. Shahcheraghi GH, Hobbi MH (1999) Patterns and pro-gression in congenital scoliosis. J Pediatr Orthop, 19: 766-775.

20. Shands AR, Eisberg HB (1955) The incidence of scoliosis in the state of Delaware. A study of 50,000 minifilms of the chest made during a survey for tuberculosis. J Bone Joint Surg, 37A: 1243.

21. Winter RB, Moe JH, Eilers VE (1968) Congenital scoliosis. A study of 234 patients treated and untreated. J Bone Joint Surg Am, 59: 15-47.

22. Zhu F, Qiu Y, Yeung HY, Lee KM, Cheng CJ (2009) Trabecular bone micro-architecture and bone mineral density in adolescent idiopathic and congenital scoliosis. Orthopaedic Surgery, 1: 78-83. 